\section{Assessment of participation bias in cohort studies: systematic review and meta- regression analysis}

\author{
Avaliação do viés de participação em estudos de \\ coorte: uma revisão sistemática e metarregressão \\ Evaluación del sesgo de participación en estudios \\ de cohortes: una revisión sistemática y \\ metarregresión
}

Sérgio Henrique Almeida da Silva Junior 1,2 Simone M. Santos 1 Cláudia Medina Coeli 3 Marilia Sá Carvalho 4

\footnotetext{
${ }^{1}$ Escola Nacional de Saúde Pública Sergio Arouca, Fundação Oswaldo Cruz, Rio de Janeiro, Brasil.

2 Instituto Benjamin

Constant, Rio de Janeiro, Brasil.

3 Instituto de Estudos em Saúde Coletiva, Universidade Federal do Rio de Janeiro, Brasil.

4 Programa de Computação Científica, Fundação Oswaldo Cruz, Rio de Janeiro, Brasil.

Correspondence S. H. A. Silva Junio Rua Jaguari 79, Nova Iguaçu, RJ 26010-425, Brasil. sergio.edfisica@gmail.com
}

\begin{abstract}
The proportion of non-participation in cohort studies, if associated with both the exposure and the probability of occurrence of the event, can introduce bias in the estimates of interest. The aim of this study is to evaluate the impact of participation and its characteristics in longitudinal studies. A systematic review (MEDLINE, Scopus and Web of Science) for articles describing the proportion of participation in the baseline of cohort studies was performed. Among the 2,964 initially identified, 50 were selected. The average proportion of participation was $64.7 \%$. Using a meta-regression model with mixed effects, only age, year of baseline contact and study region (borderline) were associated with participation. Considering the decrease in participation in recent years, and the cost of cohort studies, it is essential to gather information to assess the potential for non-participation, before committing resources. Finally, journals should require the presentation of this information in the papers.
\end{abstract}

Selection Bias; Cohort Studies; Epidemiologic Methods

\section{Resumo}

A proporção de não-participação em estudos de coorte está associada também à exposição e à probabilidade de ocorrência do evento poder gerar viés nas estimativas de interesse. O objetivo do presente trabalho é realizar uma revisão sistemática e metanálise de artigos que descrevem a participação em estudos de coorte e avaliar as características associadas à participação. Foi realizada uma revisão sistemática (MEDLINE, Scopus e Web of Science), buscando-se artigos que descrevessem a proporção de participação na linha de base de estudos de coorte. De 2.964 artigos inicialmente identificados, foram selecionados 50. Entre esses, a proporção média de participação foi de 64,7\%. Utilizando-se o modelo de metarregressão com efeitos mistos, somente a idade, ano da linha de base e a região do estudo (limítrofe) estiveram associados à participação. Considerando a diminuição na participação em anos mais recentes e o custo dos estudos de coorte, é essencial buscar informações que permitam avaliar o potencial de não-participação antes de comprometer os recursos.

Viés de Seleção; Estudos de Coortes; Métodos Epidemiológicos 


\section{Background}

Among observational studies, the advantages of prospective cohort studies are that they are able to estimate incidence measures directly and are less vulnerable to information bias. However, participation refusal at baseline or follow-up can introduce selection bias when simultaneously associated with both exposure and the outcome 1,2 As a result, the association between exposure and outcome may differ between participants and non-participants.

Morton et al. ${ }^{3}$ observed a tendency for participation in cohort studies to decrease between 1970 and 2003. As the non-participation proportion rises, vulnerability to selection bias tends to increase. Therefore, it is recommended reporting participation proportion in observational studies 4 , designing methodological studies to evaluate the impacts of non-participation and evaluating study characteristics that may influence participation 5 .

To the best of our knowledge, and in spite of its importance, no systematic evaluation of participation in observational cohort studies is available to guide choices and scientific assessment of validity of conclusions. This present study aims to perform a systematic review and meta-regression of papers describing non-participation bias in cohort studies, and evaluate the studies' characteristics associated with participation proportion.

\section{Methods}

We performed a systematic review and meta-regression following the methodology proposed by Higgins \& Green ${ }^{6}$ and PRISMA (Preferred Reporting Items for Systematic Reviews and Meta-Analyses) criteria $?$.

\section{Search strategy}

We searched MEDLINE, Scopus and Web of Science data bases for papers published between January 1978 and November 2014. The query used for the MEDLINE search strategy was: (cooperation[Title/Abstract/ MESH] or noncooperation[Title/Abstract/ MESH] or non-cooperation[Title/Abstract/ MESH] or participant*[Title/Abstract/ MESH] or nonparticipant*[Title/Abstract/ MESH] or non-participant*[Title/Abstract/ MESH] or compliance[Title/Abstract/MESH] or noncompliance[Title/Abstract/MESH] or non-compliance[Title/Abstract/MESH]) AND bias*[Title/Abstract/MESH] AND (cohort*[Title/ Abstract/MESH] OR prospective [Title/Abstract/ MESH] OR longitudinal [Title/Abstract/MESH]).
For the other data bases, the specific syntaxes corresponding to each base were used.

Article titles and abstracts were evaluated by two reviewers working independently in order to ascertain whether they met the criteria for inclusion in the study. Disagreements were assessed by a third reviewer.

\section{Eligibility criteria and data extraction}

As specific populations and health problems may induce large differences in participation proportions related to theses specificities, we only included population-based cohort studies on adult (18 to 75 years old) healthy people. We excluded studies that addressed specific populations (eg. pregnant women, patients with specific ailments), review studies and others (eg. genetic studies, surgery, drug therapies). Figure 1 depicts the review flow chart.

The references identified were stored and processed using the JabRef 2.10 software (http:// jabref.sourceforge.net/). We collected the participation proportion, the general characteristics of the study (year of baseline contact, place, selection strategy and study outcome). We also evaluated the characteristics of the study population including type (general population vs. working population), participation of women and the mean age. The relevant data was extracted reading the full paper.

\section{Data analysis}

A meta-analysis of participation proportion was conducted using mixed-effects models, often called binominal-normal models 8 . Given the heterogeneity of the studies $\left(\mathrm{I}^{2}=99.97 \% ; \tau^{2}\right.$ $=0.54 ; \mathrm{p}<0.001)$, we investigated the variables associated with the participation proportion, initially by simple meta-regression models. When the value of variance accounted for (VAF) by the model was greater than $5 \%$, the variable was included in the multiple model. VAF indicates the percentage of total heterogeneity that is explained by each moderator. The goodness of fit of the multiple model was evaluated by the likelihood ratio test (LRT).

We analyzed the following variables: year of the baseline contact, participant mean age, proportion of women, selection strategy, population type (general population vs. employees population), study outcome - cardiovascular (baseline category), general health or others (cancer, accident, substance use, incapacity and smoking) - and study region, as divided by United Nations Statistics Division 9 into Continental Europe (baseline category), Northern Europe, USA, and 
Others (Asia or Oceania). Spearman correlation coefficient was used to evaluate the relation between the year of the baseline contact and the participation proportion.

The analyses were performed using the metafor 10 library of R software (The R Foundation for Statistical Computing, Vienna, Austria; http:// www.r-project.org).

\section{Results}

Of the 2,964 original papers initially identified, 50 were selected. Figure 1 summarizes the study selection process.

Table 1 describes the objectives, database, analysis and main results of the selected papers. To evaluate participation, 29 (58\%) papers compared participants and non-participants using secondary databases, 15 (30\%) used the information available at baseline, and six (12\%) used some way of contacting the non-respondents with small questionnaires. Logistic regression models were the most used technique to evaluate participation, used in 18 (40\%) of the papers. Passive follow-up studies applied survival (7) and Poisson regression models (4), and a few some combination of different techniques. In eight papers the evaluation was based on frequencies comparison, using baseline characteristics and/or questionnaires. Imputation, weighted regression and simulations were applied in four papers to evaluate and propose analytical methods for correcting potential bias.

Table 2 describes of the overall study characteristics and sample characteristics potentially associated with participation proportion. Most of the publications are concentrated in the years from 2005 to 2014, the oldest having been published in 1978. The studies comprised 40 (80\%) geographically population-based, while the remainder were of workers (8), students (1) and recruits (1).

Most of the studies were conducted in Northern Europe (40\%). Regarding participant selection, $60 \%$ were random sample, the remainder census-based. The most frequent outcomes were overall health condition in twenty-three (46\%), and cardiovascular health in forteen. Other outcomes included cancer, accident, substance use, incapacity and smoking. Participant mean age was 49.5 years ( $\mathrm{SD}=8.2$ years). Mean participation proportion was $64.7 \%$, and ranged from $32.2 \%$ to $87.3 \%$. Women participation was slightly larger (52.6\%) (Table 2).

A negative correlation was found between study year and participation proportion ( $\rho=$ -0.38). Figure 2 shows the downward trend in participation proportion. The dotted line indicates the linear regression, an annual rate of decrease of $0.66 \%\left(R^{2}=0.1 ; p=0.01\right)$. The continuous line (a smooth spline) indicates a downward trend in participation, since 1985 . The diameters of the circles of each study, identified by the number of the study (id) in Table 1, is proportional to the inverse of the corresponding standard errors in the meta-regression. The larger circles are more influential in the meta-regression.

The simple meta-regression showed association only between participation proportion and year of the baseline contact $(\mathrm{OR}=0.97$; 95\%CI: 0.95-0.99). The multiple meta-regression showed an association between participation proportion, year of the baseline contact $(\mathrm{OR}=0.97 ; 95 \% \mathrm{CI}$ : 0.95-0.99) and age (OR = 0.97; 95\%CI: 0.95-1.00) (Table 3). In other words, for one-year increase in the year of the baseline contact of the study we expect a $3 \%$ decrease in the odds of study participation. Likewise, for one-year increase in the mean age of the study participants we expect a $3 \%$ reduction in the odds of study participation.

The analysis shows residual heterogeneity $\tau^{2}=0.41(\mathrm{p}<0.001)$ for the participation proportion, suggesting that $18.1 \%$ of total heterogeneity can be accounted for by including year of the baseline contact and age. The test for residual heterogeneity is significant (LRT $=42,252.5, \mathrm{df}=33, \mathrm{p}=$ 0.00 ), indicating that other covariates not considered in the model are influencing the participation proportion.

\section{Discussion}

We found a high heterogeneity in participation proportions among the papers evaluating nonparticipation bias. The most referred characteristics described in the systematic reviewed papers were sociodemographic profile, hospitalization and cancer incidence. Mortality was larger among non-participants. However, in the meta-regression performed only year of the baseline contact and age was associated with participation.

Several strategies involving comparison between participants and non-participants have been proposed to evaluate the potential selection bias in cohort studies: questionnaires to non-participants, comparison of participants according to recruitment moment 4 and passive monitoring of the eligible population using secondary database to assess the outcome 11, the majority of papers in our study.

The results show a decrease in participation in studies over time. The reasons for this decline are not clear, but social changes, and changes in selection and recruitment and in study designs may influence participation ${ }^{3}$. The decrease in participa- 
Flowchart of the search and selection of studies included in the meta-analysis.

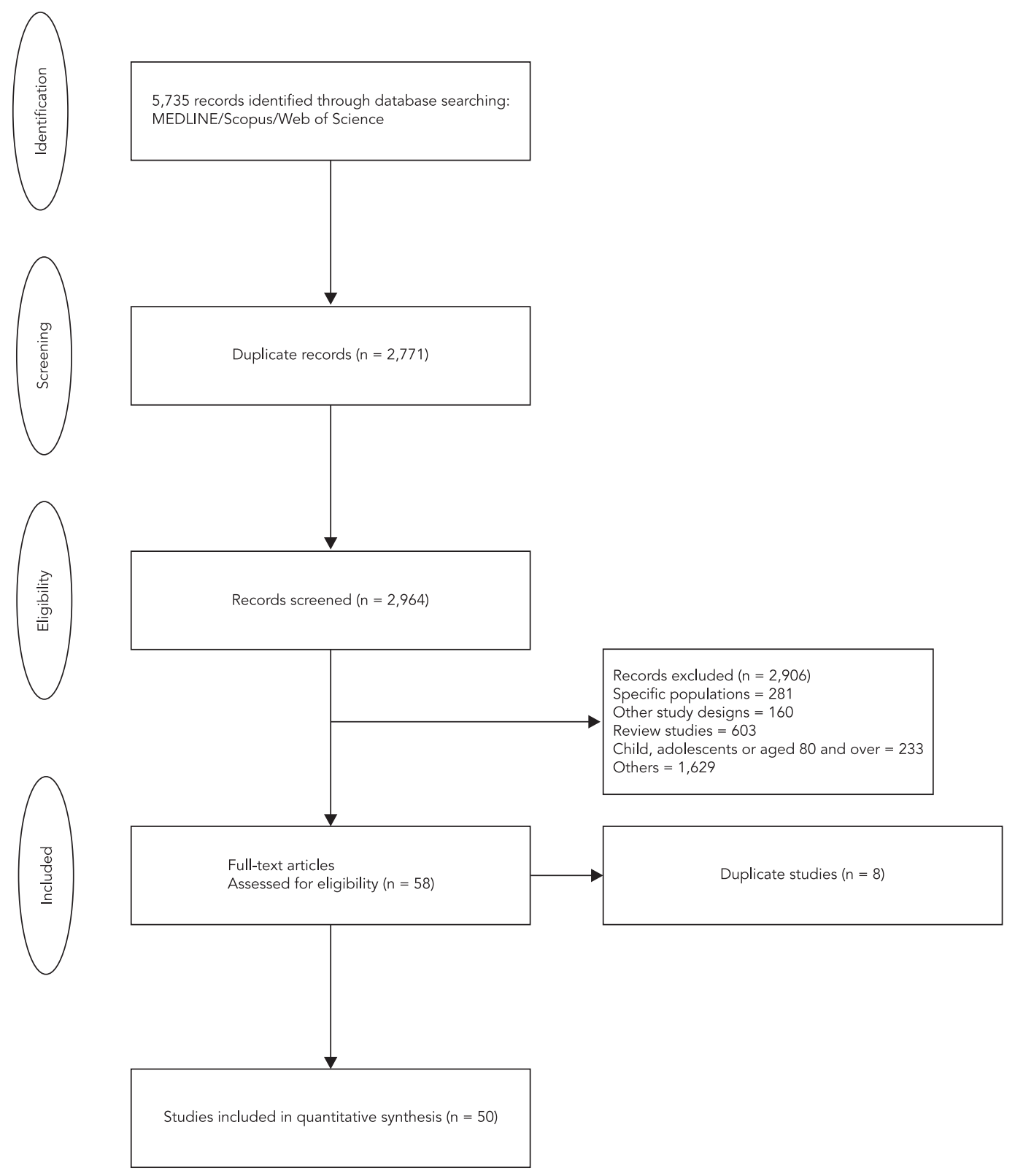

Source: Moher et al. 7 .

tion may be related particularly to the increasing number of studies in recent decades, as well as the proliferation of political and marketing surveys 5 . In addition, increased requests for biological material in epidemiological studies may influence adherence negatively 3 .
Previous studies have reported the association between young age and participation cohort studies. Contrary to other articles 12,13,14,15 the proportion of women in the studies showed no association with participation, not even in the simple model. The outcome of the studies was not associ- 
Table 1

Characteristics of studies potentially associated with participation.

\begin{tabular}{|c|c|c|c|c|c|c|c|c|c|}
\hline Id & Reference & $\begin{array}{c}\text { Baseline } \\
\text { year }\end{array}$ & Source population & Study region & Outcome & Selection & Mean age & $\mathrm{N}$ & Participation rate (\%) \\
\hline 1 & Studer et al. 37 & 2010 & Recruits & $\begin{array}{l}\text { Continental } \\
\text { Europe }\end{array}$ & Other & Sampling & 20 & 5,457 & Overall $=46.2$ \\
\hline 2 & Kaerlev et al. 38 & 2007 & Workers in general & $\begin{array}{l}\text { Northern } \\
\text { Europe }\end{array}$ & General health & Sampling & 45 & 4,489 & $\begin{array}{c}\text { Overall }=44.7 ; \text { Men }= \\
\text { 79.2; } \text { Women }=20.8\end{array}$ \\
\hline 3 & Langley et al. 39 & 2007 & $\begin{array}{c}\text { General } \\
\text { population }\end{array}$ & Other & Other & Sampling & 41.4 & 2,856 & $\begin{array}{c}\text { Overall }=79.9 ; \text { Men }= \\
61.0 ; \text { Women }=39.0\end{array}$ \\
\hline 4 & Alkerwi et al. 40 & 2006 & $\begin{array}{c}\text { General } \\
\text { population }\end{array}$ & $\begin{array}{l}\text { Continental } \\
\text { Europe }\end{array}$ & $\begin{array}{c}\text { Cardiovascular } \\
\text { diseases }\end{array}$ & Sampling & 44.3 & 1,432 & $\begin{array}{c}\text { Overall }=32.2 ; \text { Men }= \\
48.7 ; \text { Women }=51.3\end{array}$ \\
\hline 5 & $\begin{array}{c}\text { Langhammer et } \\
\text { al. } 41\end{array}$ & 2006 & $\begin{array}{c}\text { General } \\
\text { population }\end{array}$ & $\begin{array}{l}\text { Northern } \\
\text { Europe }\end{array}$ & General health & Census & 53.1 & 50,807 & $\begin{array}{c}\text { Overall }=54.1 ; \text { Men }= \\
45.4 ; \text { Women }=54.6\end{array}$ \\
\hline 6 & $\begin{array}{l}\text { Eriksson et } \\
\text { al. } 42\end{array}$ & 2005 & $\begin{array}{c}\text { General } \\
\text { population }\end{array}$ & $\begin{array}{l}\text { Northern } \\
\text { Europe }\end{array}$ & $\begin{array}{c}\text { Cardiovascular } \\
\text { diseases }\end{array}$ & Census & 47 & 25,173 & $\begin{array}{c}\text { Overall }=82.6 ; \text { Men = } \\
39.4 ; \text { Women }=60.6\end{array}$ \\
\hline 7 & Osler et al. 43 & 2004 & $\begin{array}{c}\text { General } \\
\text { population }\end{array}$ & $\begin{array}{l}\text { Northern } \\
\text { Europe }\end{array}$ & General health & Census & 51 & 6,292 & Overall $=66.2$ \\
\hline 8 & Buckley et al. 44 & 2003 & $\begin{array}{l}\text { General } \\
\text { population }\end{array}$ & $\begin{array}{l}\text { Northern } \\
\text { Europe }\end{array}$ & $\begin{array}{c}\text { Cardiovascular } \\
\text { diseases }\end{array}$ & Sampling & 63.9 & 493 & $\begin{array}{c}\text { Overall }=45.6 ; \text { Men }= \\
80.9 ; \text { Women }=19.1\end{array}$ \\
\hline 9 & $\begin{array}{l}\text { Schmidt et } \\
\text { al. } 45\end{array}$ & 2003 & $\begin{array}{c}\text { General } \\
\text { population }\end{array}$ & $\begin{array}{l}\text { Continental } \\
\text { Europe }\end{array}$ & General health & Sampling & 46.7 & 7,189 & $\begin{array}{c}\text { Overall }=64.5 ; \text { Men }= \\
45.7 ; \text { Women }=54.3\end{array}$ \\
\hline 10 & $\begin{array}{c}\text { Martikainen et } \\
\text { al. } 46\end{array}$ & 2002 & Workers in general & $\begin{array}{l}\text { Northern } \\
\text { Europe }\end{array}$ & General health & Sampling & 49.6 & 8,960 & Overall = 67.1 \\
\hline 11 & Holden et al. 47 & 2001 & $\begin{array}{c}\text { General } \\
\text { population }\end{array}$ & Other & General health & Census & 65 & 1,115 & $\begin{array}{c}\text { Overall }=42.6 ; \text { Men }= \\
\text { 49.9; } \text { Women }=50.1\end{array}$ \\
\hline 12 & Lissner et al. 17 & 2001 & $\begin{array}{c}\text { General } \\
\text { population }\end{array}$ & $\begin{array}{l}\text { Northern } \\
\text { Europe }\end{array}$ & General health & Sampling & 46.8 & 850 & Overall $=71$ \\
\hline 13 & Stang et al. 16 & 2001 & $\begin{array}{c}\text { General } \\
\text { population }\end{array}$ & $\begin{array}{l}\text { Continental } \\
\text { Europe }\end{array}$ & $\begin{array}{c}\text { Cardiovascular } \\
\text { diseases }\end{array}$ & Sampling & 58.8 & 8,413 & $\begin{array}{c}\text { Overall }=53.3 ; \text { Men }= \\
54.3 ; \text { Women }=45.7\end{array}$ \\
\hline 14 & $\begin{array}{l}\text { Goldberg et } \\
\text { al. } 20\end{array}$ & 2000 & $\begin{array}{l}\text { Electric and gas } \\
\text { utility workers }\end{array}$ & $\begin{array}{l}\text { Continental } \\
\text { Europe }\end{array}$ & General health & Census & 45.1 & 20,328 & $\begin{array}{c}\text { Overall }=44.1 ; \text { Men }= \\
\text { 72.9; } \text { Women }=27.1\end{array}$ \\
\hline 15 & Taylor et al. 48 & 2000 & $\begin{array}{c}\text { General } \\
\text { population }\end{array}$ & Other & General health & Sampling & 46 & 6,073 & $\begin{array}{c}\text { Overall }=49.6 ; \text { Men }= \\
\text { 48.9; } \text { Women }=51.1\end{array}$ \\
\hline 16 & Alonso et al. 49 & 1999 & Students & $\begin{array}{l}\text { Continental } \\
\text { Europe }\end{array}$ & $\begin{array}{c}\text { Cardiovascular } \\
\text { diseases }\end{array}$ & Census & 35.4 & 9,907 & $\begin{array}{c}\text { Overall }=87.3 ; \text { Men }= \\
40.7 ; \text { Women }=59.3\end{array}$ \\
\hline 17 & $\begin{array}{l}\text { Knudsen et } \\
\text { al. } 19\end{array}$ & 1999 & $\begin{array}{c}\text { General } \\
\text { population }\end{array}$ & $\begin{array}{l}\text { Northern } \\
\text { Europe }\end{array}$ & $\begin{array}{c}\text { Cardiovascular } \\
\text { diseases }\end{array}$ & Census & 48.8 & 18,565 & $\begin{array}{c}\text { Overall }=63.2 ; \text { Men }= \\
51.2 ; \text { Women }=48.8\end{array}$ \\
\hline 18 & Manjer et al. 29 & 1999 & $\begin{array}{c}\text { General } \\
\text { population }\end{array}$ & $\begin{array}{l}\text { Northern } \\
\text { Europe }\end{array}$ & Other & Census & 52.9 & 28,098 & $\begin{array}{c}\text { Overall }=60.5 ; \text { Men }= \\
39.4 ; \text { Women }=60.6\end{array}$ \\
\hline 19 & $\begin{array}{l}\text { Barchielli \& } \\
\text { Balzi } 24\end{array}$ & 1998 & $\begin{array}{c}\text { General } \\
\text { population }\end{array}$ & $\begin{array}{l}\text { Continental } \\
\text { Europe }\end{array}$ & Other & Sampling & 61.8 & 1,776 & $\begin{array}{c}\text { Overall }=85.8 ; \text { Men }= \\
44.3 ; \text { Women }=55.7\end{array}$ \\
\hline 20 & $\begin{array}{l}\text { Bergman et } \\
\text { al. } 50\end{array}$ & 1998 & $\begin{array}{c}\text { General } \\
\text { population }\end{array}$ & $\begin{array}{l}\text { Northern } \\
\text { Europe }\end{array}$ & Other & Census & 42.7 & 19,742 & $\begin{array}{c}\text { Overall }=52.9 ; \text { Men }= \\
44.5 ; \text { Women }=55.5\end{array}$ \\
\hline 21 & $\begin{array}{l}\text { Petersen et } \\
\text { al. } 51\end{array}$ & 1998 & $\begin{array}{c}\text { General } \\
\text { population }\end{array}$ & $\begin{array}{l}\text { Northern } \\
\text { Europe }\end{array}$ & Other & Census & 63.9 & 791 & $\begin{array}{c}\text { Overall }=38.4 ; \text { Men }= \\
41.8 ; \text { Women }=58.2\end{array}$ \\
\hline 22 & Rao et al. 52 & 1998 & Radiologists & U.S.A. & Other & Census & 50.1 & 90,305 & Overall $=68.4$ \\
\hline 23 & Haring et al. 53 & 1997 & $\begin{array}{c}\text { General } \\
\text { population }\end{array}$ & $\begin{array}{l}\text { Continental } \\
\text { Europe }\end{array}$ & General health & Sampling & 54.1 & 7,008 & $\begin{array}{c}\text { Overall }=47.1 ; \text { Men }= \\
48.2 ; \text { Women }=51.8\end{array}$ \\
\hline 24 & $\begin{array}{l}\text { Van Loon et } \\
\text { al. } 30\end{array}$ & 1997 & $\begin{array}{c}\text { General } \\
\text { population }\end{array}$ & $\begin{array}{l}\text { Continental } \\
\text { Europe }\end{array}$ & General health & Sampling & 42.2 & 12,097 & $\begin{array}{c}\text { Overall }=56.5 ; \text { Men }= \\
44.5 ; \text { Women }=55.5\end{array}$ \\
\hline 25 & $\begin{array}{l}\text { Drivsholm et } \\
\text { al. } 54\end{array}$ & 1996 & $\begin{array}{l}\text { General } \\
\text { population }\end{array}$ & $\begin{array}{l}\text { Northern } \\
\text { Europe }\end{array}$ & Other & Census & 60 & 1,077 & $\begin{array}{c}\text { Overall }=64.5 ; \text { Men }= \\
46.8 ; \text { Women }=53.2\end{array}$ \\
\hline
\end{tabular}

(continues) 


\begin{tabular}{|c|c|c|c|c|c|c|c|c|c|}
\hline Id & Reference & $\begin{array}{c}\text { Baseline } \\
\text { year }\end{array}$ & Source population & Study region & Outcome & Selection & Mean age & $\mathbf{N}$ & Participation rate (\%) \\
\hline 26 & $\begin{array}{c}\text { Jackson et } \\
\text { al. } 15\end{array}$ & 1996 & $\begin{array}{c}\text { General } \\
\text { population }\end{array}$ & U.S.A. & $\begin{array}{c}\text { Cardiovascular } \\
\text { diseases }\end{array}$ & Sampling & 54 & 15,800 & $\begin{array}{c}\text { Overall = 59.8; } \text { Men }= \\
45.1 ; \text { Women }=54.9\end{array}$ \\
\hline 27 & $\begin{array}{c}\text { Veenstra et } \\
\text { al. } 28\end{array}$ & 1996 & $\begin{array}{c}\text { General } \\
\text { population }\end{array}$ & $\begin{array}{l}\text { Continental } \\
\text { Europe }\end{array}$ & $\begin{array}{c}\text { Cardiovascular } \\
\text { diseases }\end{array}$ & Sampling & 55.8 & 15,896 & $\begin{array}{c}\text { Overall = 51.9; } \text { Men }= \\
50.5 ; \text { Women }=49.5\end{array}$ \\
\hline 28 & Young et al. 55 & 1996 & $\begin{array}{c}\text { General } \\
\text { population }\end{array}$ & Other & General health & Sampling & 47.5 & 40,395 & Overall $=80.4$ \\
\hline 29 & $\begin{array}{c}\text { Caetano et } \\
\text { al. } 56\end{array}$ & 1995 & $\begin{array}{c}\text { General } \\
\text { population }\end{array}$ & U.S.A. & Other & Sampling & 42.2 & 3,106 & $\begin{array}{c}\text { Overall = 81.8; } \text { Men = } \\
48.2 ; \text { Women }=51.8\end{array}$ \\
\hline 30 & Garcia et al. 27 & 1994 & $\begin{array}{c}\text { General } \\
\text { population }\end{array}$ & $\begin{array}{c}\text { Continental } \\
\text { Europe }\end{array}$ & General health & Sampling & 39.1 & 1,438 & $\begin{array}{c}\text { Overall = 57.5; Men = } \\
46.5 ; \text { Women }=53.5\end{array}$ \\
\hline 31 & Hara et al. 57 & 1994 & $\begin{array}{c}\text { General } \\
\text { population }\end{array}$ & Other & Other & Sampling & 55.5 & 61,447 & $\begin{array}{c}\text { Overall = 50.5; Men = } \\
46.7 ; \text { Women }=53.8\end{array}$ \\
\hline 32 & $\begin{array}{c}\text { Kjøller \& } \\
\text { Thoning } 32\end{array}$ & 1994 & $\begin{array}{c}\text { General } \\
\text { population }\end{array}$ & $\begin{array}{l}\text { Northern } \\
\text { Europe }\end{array}$ & General health & Sampling & 45.6 & 18,292 & $\begin{array}{c}\text { Overall = 79.2; } \text { Men = } \\
48.5 ; \text { Women }=51.5\end{array}$ \\
\hline 33 & $\begin{array}{c}\text { Jacobsen et } \\
\text { al. } 31\end{array}$ & 1993 & $\begin{array}{c}\text { General } \\
\text { population }\end{array}$ & U.S.A. & $\begin{array}{l}\text { Cardiovascular } \\
\text { diseases }\end{array}$ & Sampling & 60.8 & 963 & $\begin{array}{c}\text { Overall = 50.6; } \text { Men = } \\
47.3 ; \text { Women }=52.7\end{array}$ \\
\hline 34 & $\begin{array}{c}\text { Montgomery et } \\
\text { al. } 58\end{array}$ & 1993 & $\begin{array}{c}\text { Pesticide } \\
\text { applicators }\end{array}$ & U.S.A. & General health & Census & 47.3 & 50,764 & $\begin{array}{c}\text { Overall = 65.9; } \text { Men = } \\
97.0 ; \text { Women }=3.0\end{array}$ \\
\hline 35 & $\begin{array}{c}\text { Jousilahti et } \\
\text { al. } 59\end{array}$ & 1992 & $\begin{array}{c}\text { General } \\
\text { population }\end{array}$ & $\begin{array}{l}\text { Northern } \\
\text { Europe }\end{array}$ & General health & Sampling & 48.1 & 6,051 & $\begin{array}{c}\text { Overall = 84.4; } \text { Men = } \\
47.1 ; \text { Women }=52,9\end{array}$ \\
\hline 36 & May et al. 60 & 1992 & $\begin{array}{c}\text { General } \\
\text { population }\end{array}$ & Other & General health & Sampling & 52.2 & 375,815 & $\begin{array}{c}\text { Overall }=81.6 ; \text { Men }= \\
27.6 ; \text { Women }=72.4\end{array}$ \\
\hline 37 & Batty \& Gale 61 & 1991 & $\begin{array}{c}\text { General } \\
\text { population }\end{array}$ & $\begin{array}{l}\text { Northern } \\
\text { Europe }\end{array}$ & $\begin{array}{c}\text { Cardiovascular } \\
\text { diseases }\end{array}$ & Sampling & 51 & 6,484 & $\begin{array}{c}\text { Overall = 70.8; } \text { Men = } \\
44.7 ; \text { Women }=55.3\end{array}$ \\
\hline 38 & Dugué et al. 62 & 1990 & $\begin{array}{c}\text { General } \\
\text { population }\end{array}$ & $\begin{array}{l}\text { Northern } \\
\text { Europe }\end{array}$ & General health & Census & 33.2 & $1,156,671$ & Overall = 78.1 \\
\hline 39 & Hara et al. 22 & 1990 & $\begin{array}{c}\text { General } \\
\text { population }\end{array}$ & Other & $\begin{array}{c}\text { Cardiovascular } \\
\text { diseases }\end{array}$ & Census & 49.6 & 43,140 & $\begin{array}{c}\text { Overall = 79.3; Men = } \\
\text { 48.0; Women }=52.0\end{array}$ \\
\hline 40 & $\begin{array}{c}\text { Benfante et } \\
\text { al. } 63\end{array}$ & 1989 & $\begin{array}{c}\text { General } \\
\text { population }\end{array}$ & U.S.A. & $\begin{array}{c}\text { Cardiovascular } \\
\text { diseases }\end{array}$ & Census & 54.3 & 8,006 & Overall = 71.9 \\
\hline 41 & Ferrie et al. 23 & 1988 & Office workers & $\begin{array}{l}\text { Northern } \\
\text { Europe }\end{array}$ & General health & Census & 46.3 & 10,297 & $\begin{array}{c}\text { Overall = 87.1; Men = } \\
67.0 ; \text { Women }=33.0\end{array}$ \\
\hline 42 & $\begin{array}{c}\text { François et } \\
\text { al. } 64\end{array}$ & 1987 & $\begin{array}{c}\text { General } \\
\text { population }\end{array}$ & $\begin{array}{l}\text { Continental } \\
\text { Europe }\end{array}$ & Other & Sampling & 43,3 & 1,910 & $\begin{array}{c}\text { Overall = 83.1; } \text { Men = } \\
48.9 ; \text { Women }=51.1\end{array}$ \\
\hline 43 & Walker et al. 21 & 1987 & $\begin{array}{c}\text { General } \\
\text { population }\end{array}$ & $\begin{array}{l}\text { Northern } \\
\text { Europe }\end{array}$ & $\begin{array}{c}\text { Cardiovascular } \\
\text { diseases }\end{array}$ & Sampling & 46.4 & 15,364 & Overall $=74.3$ \\
\hline 44 & David et al. 65 & 1986 & $\begin{array}{c}\text { General } \\
\text { population }\end{array}$ & Other & Other & Sampling & 48.7 & 2,095 & $\begin{array}{c}\text { Overall = 78.0; Men = } \\
\text { 43.8; } \text { Women }=56.2\end{array}$ \\
\hline 45 & Froom et al. 66 & 1985 & $\begin{array}{l}\text { Industrial } \\
\text { employees }\end{array}$ & Other & General health & Census & 45 & 5,302 & Overall $=71.6$ \\
\hline 46 & Bopp et al. 67 & 1984 & $\begin{array}{c}\text { General } \\
\text { population }\end{array}$ & $\begin{array}{l}\text { Continental } \\
\text { Europe }\end{array}$ & $\begin{array}{c}\text { Cardiovascular } \\
\text { diseases }\end{array}$ & Census & 47.6 & 10,160 & $\begin{array}{c}\text { Overall }=33.9 ; \text { Men }= \\
\text { 49.1; Women } 50.9\end{array}$ \\
\hline 47 & Criqui et al. 68 & 1978 & $\begin{array}{c}\text { General } \\
\text { population }\end{array}$ & U.S.A. & $\begin{array}{c}\text { Cardiovascular } \\
\text { diseases }\end{array}$ & Sampling & 52.5 & 5,052 & $\begin{array}{c}\text { Overall = 82.1; } \text { Men = } \\
46.0 ; \text { Women }=54.0\end{array}$ \\
\hline 48 & $\begin{array}{l}\text { Lindsted et } \\
\text { al. } 69\end{array}$ & 1976 & $\begin{array}{c}\text { General } \\
\text { population }\end{array}$ & U.S.A. & General health & Census & 53 & 39,886 & $\begin{array}{c}\text { Overall = 78.0; } \text { Men }= \\
40.8 ; \text { Women }=59.2\end{array}$ \\
\hline 49 & $\begin{array}{l}\text { Thygesen et } \\
\text { al. } 70\end{array}$ & 1976 & $\begin{array}{c}\text { General } \\
\text { population }\end{array}$ & $\begin{array}{l}\text { Northern } \\
\text { Europe }\end{array}$ & General health & Sampling & 53.1 & 24,464 & $\begin{array}{c}\text { Overall = 72.0; } \text { Men }= \\
45.8 ; \text { Women }=54.2\end{array}$ \\
\hline 50 & $\begin{array}{c}\text { Vestbo \& } \\
\text { Rasmussen } 71\end{array}$ & 1974 & Workers in general & $\begin{array}{l}\text { Northern } \\
\text { Europe }\end{array}$ & Other & Sampling & 55.1 & 1,404 & Overall = 66.1 \\
\hline
\end{tabular}




\section{Table 2}

Objectives, database, analysis and results of the selected papers.

\begin{tabular}{|c|c|c|c|c|c|}
\hline Id & Reference & Objectives * & Data source & Analysis & Results \\
\hline 1 & Studer et al. 38 & $\begin{array}{l}\text { To evaluate differences in substance } \\
\text { use between late and early } \\
\text { respondents, non-consenters or silent } \\
\text { refusers, and whether converting } \\
\text { former non-respondents can reduce } \\
\text { non-response bias }\end{array}$ & $\begin{array}{l}\text { Baseline } \\
\text { information }\end{array}$ & Logistic model & $\begin{array}{l}\text { Late respondents presented a midway pattern of } \\
\text { substance use higher than early respondents, but } \\
\text { lower than non-consenters }\end{array}$ \\
\hline 2 & Kaerlev et al. 39 & $\begin{array}{l}\text { To examine bias on the association } \\
\text { between occupational stressors and } \\
\text { mental health due to non-participation } \\
\text { in a prospective cohort }\end{array}$ & Secondary data & Survival model & $\begin{array}{l}\text { Proportions of gender, age, employment status, sick } \\
\text { leave and hospitalization for affective disorders were } \\
\text { different in respondents and non-respondents, but } \\
\text { low participation at baseline was not associated with } \\
\text { mental health outcome }\end{array}$ \\
\hline 3 & Langley et al. 40 & $\begin{array}{l}\text { To evaluate factors associated with } \\
\text { non-participation in two follow-up } \\
\text { contacts of a prospective cohort study } \\
\text { of injury outcomes }\end{array}$ & $\begin{array}{l}\text { Baseline } \\
\text { information }\end{array}$ & Poisson model & $\begin{array}{l}\text { Non-participation in the closest follow-up contact } \\
\text { did not mean non-participation in the next contact; } \\
\text { sociodemographic factors were the most important } \\
\text { for non-participation }\end{array}$ \\
\hline 4 & Alkerwi et al. 41 & $\begin{array}{l}\text { To evaluate the representativeness } \\
\text { of the sample with respect to } \\
\text { the population and compare } \\
\text { characteristics of participants and non } \\
\text { participants }\end{array}$ & $\begin{array}{l}\text { Baseline } \\
\text { information }\end{array}$ & Logistic model & $\begin{array}{l}\text { Non-participants were similar to participants in } \\
\text { gender and place of residence; younger people were } \\
\text { under-represented while adults and elderly were over- } \\
\text { represented; no discriminating health profiles were } \\
\text { detected }\end{array}$ \\
\hline 5 & $\begin{array}{l}\text { Langhammer et } \\
\qquad \text { al. } 42\end{array}$ & $\begin{array}{l}\text { To study potential participation bias } \\
\text { for common symptoms, diseases and } \\
\text { socioeconomic status and mortality by } \\
\text { participation status }\end{array}$ & $\begin{array}{l}\text { Secondary } \\
\text { data, mailed } \\
\text { questionnaire. }\end{array}$ & $\begin{array}{l}\text { Negative } \\
\text { binomial and } \\
\text { survival models }\end{array}$ & $\begin{array}{l}\text { Questionnaire answers indicated higher prevalences } \\
\text { of cardiovascular diseases, diabetes mellitus and } \\
\text { psychiatric disorders among non-participants; } \\
\text { registry data showed higher mortality and lower } \\
\text { socioeconomic status among non-participants }\end{array}$ \\
\hline 6 & Eriksson et al. 43 & $\begin{array}{l}\text { To assess selective non-response in } \\
\text { population-based cohort study on } \\
\text { type } 2 \text { diabetes, using the population- } \\
\text { based drug register for the Stockholm } \\
\text { Diabetes Prevention Program }\end{array}$ & Secondary data & Logistic model & $\begin{array}{l}\text { At baseline, non-participants and participants were } \\
\text { similar. At follow-up, risks were higher among non- } \\
\text { participants }\end{array}$ \\
\hline 7 & Osler et al. 44 & $\begin{array}{l}\text { To evaluate changes in association } \\
\text { measures in early-life aspects and later } \\
\text { health outcomes due to non-response } \\
\text { in a follow-up survey }\end{array}$ & Secondary data & $\begin{array}{l}\text { Logistic } \\
\text { model and } \\
\text { comparison } \\
\text { of odds ratios } \\
\text { between } \\
\text { respondents } \\
\text { and complete } \\
\text { cohort }\end{array}$ & $\begin{array}{l}\text { A low response rate at age } 50 \text { years was related } \\
\text { to having a single mother at birth, low educational } \\
\text { attainment at age } 18 \text {, and low cognitive function } \\
\text { at ages } 12 \text { and } 18 \text {. The risk of alcohol overuse and } \\
\text { tobacco-related diseases was also highest among } \\
\text { non-respondents }\end{array}$ \\
\hline 8 & Buckley et al. 45 & $\begin{array}{l}\text { To assess baseline differences in } \\
\text { participation in a secondary prevention } \\
\text { of ischemic heart disease program }\end{array}$ & Secondary data & Logistic model & $\begin{array}{l}\text { Enrollment was lower for women in general and for } \\
\text { men with uncontrolled total cholesterol level }\end{array}$ \\
\hline 9 & Schmidt et al. 46 & $\begin{array}{l}\text { To identify back-pain-related indicators } \\
\text { that could predict attrition in } \\
\text { longitudinal studies }\end{array}$ & $\begin{array}{l}\text { Baseline } \\
\text { information }\end{array}$ & Logistic model & $\begin{array}{l}\text { The best predictors of attrition were age and baseline } \\
\text { response behavior. No bias was found in relation to } \\
\text { back pain indicators }\end{array}$ \\
\hline 10 & $\begin{array}{l}\text { Martikainen et } \\
\text { al. } 47\end{array}$ & $\begin{array}{l}\text { To estimate impact on social class } \\
\text { inequalities in health due to non- } \\
\text { response }\end{array}$ & Secondary data & $\begin{array}{l}\text { Linear } \\
\text { regression } \\
\text { model }\end{array}$ & $\begin{array}{l}\text { Higher social class employees and women were more } \\
\text { likely to participate, and sickness absence was higher } \\
\text { in non-respondents. Social classes differences did } \\
\text { not impact sickness absence in participants or non- } \\
\text { participants }\end{array}$ \\
\hline
\end{tabular}

(continues) 
Table 2 (continued)

\begin{tabular}{|c|c|c|c|c|c|}
\hline Id & Reference & Objectives * & Data source & Analysis & Results \\
\hline 11 & Holden et al. 48 & $\begin{array}{l}\text { To explore reasons for non- } \\
\text { participation in a chronic disease } \\
\text { management program }\end{array}$ & Secondary data & $\begin{array}{l}\text { Logistic and } \\
\text { multinomial } \\
\text { model }\end{array}$ & $\begin{array}{l}\text { Reasons for loss-to-follow-up were: refusals - related } \\
\text { to older age, female gender and heart failure; } \\
\text { untraceable people - younger, single, indigenous; } \\
\text { and death - older individuals, male, who had cancer } \\
\text { or heart failure }\end{array}$ \\
\hline 12 & Lissner et al. 18 & $\begin{array}{l}\text { To describe } 32 \text { years of follow-up of } \\
\text { a cohort of women receiving several } \\
\text { health examinations }\end{array}$ & $\begin{array}{l}\text { Baseline } \\
\text { information, } \\
\text { home visits to } \\
\text { non-respondents }\end{array}$ & $\begin{array}{l}\text { Linear } \\
\text { regression } \\
\text { model }\end{array}$ & $\begin{array}{c}\text { Among the } 64 \% \text { of survivors, non-participants and } \\
\text { home visited subjects were similar in regard to } \\
\text { anthropometry and blood pressure, and both groups } \\
\text { were similar to participants in social indicators }\end{array}$ \\
\hline 13 & Stang et al. 17 & $\begin{array}{l}\text { To compare recruitment strategies and } \\
\text { baseline characteristics of participants } \\
\text { and non-participants }\end{array}$ & $\begin{array}{c}\text { Sample of the } \\
\text { population }\end{array}$ & $\begin{array}{l}\text { Frequencies } \\
\text { comparison }\end{array}$ & $\begin{array}{c}\text { Nonparticipants were more often smokers and of } \\
\text { lower social class. A regular relationship with a partner } \\
\text { was more frequent among participants }\end{array}$ \\
\hline 14 & $\begin{array}{c}\text { Goldberg et } \\
\text { al. } 21\end{array}$ & $\begin{array}{l}\text { To evaluate several variables } \\
\text { associated with participation in the } \\
\text { French GAZEL cohort }\end{array}$ & $\begin{array}{c}\text { Baseline } \\
\text { information }\end{array}$ & $\begin{array}{l}\text { Mixed effects } \\
\text { logistic model }\end{array}$ & $\begin{array}{l}\text { Male and older employees in managerial position or } \\
\text { retired presented higher response rates. Smoking and } \\
\text { alcohol drinking predicted lower participation. Health } \\
\text { problems were strong predictors of attrition }\end{array}$ \\
\hline 15 & Taylor et al. 49 & $\begin{array}{l}\text { To analyze the association between } \\
\text { health-related and socio-demographic } \\
\text { indicators and participation in a } \\
\text { biomedical cohort study }\end{array}$ & $\begin{array}{c}\text { Sample of the } \\
\text { population }\end{array}$ & $\begin{array}{l}\text { Frequencies } \\
\text { comparison }\end{array}$ & $\begin{array}{l}\text { Cohort participants were similar to the source } \\
\text { population, except for alcohol consumption, which, at } \\
\text { an intermediate to high risk level was more frequent } \\
\text { among participants }\end{array}$ \\
\hline 16 & Alonso et al. 50 & $\begin{array}{l}\text { To evaluate potential predictors } \\
\text { of retention in a cohort study and } \\
\text { selection bias effect in rate ratio } \\
\text { estimates due to loss-to-follow-up }\end{array}$ & $\begin{array}{c}\text { Baseline } \\
\text { information }\end{array}$ & $\begin{array}{l}\text { Inverse } \\
\text { probability } \\
\text { weight logistic } \\
\text { model }\end{array}$ & $\begin{array}{l}\text { Several variables (age, smoking, marital status, } \\
\text { obesity, past vehicle injury and self-reported history } \\
\text { of cardiovascular disease) were associated with } \\
\text { the probability of attrition. Obesity, when adjusted } \\
\text { for confounding, was similarly associated with } \\
\text { hypertension in models with and without inverse } \\
\text { probability weight }\end{array}$ \\
\hline 17 & $\begin{array}{c}\text { Knudsen et } \\
\text { al. } 20\end{array}$ & $\begin{array}{l}\text { To evaluate characteristics such as } \\
\text { health status and specific health } \\
\text { problems of non-participants in } \\
\text { population-based study, and the } \\
\text { potential resulting bias in association } \\
\text { measures }\end{array}$ & Secondary data & $\begin{array}{c}\text { Survival model, } \\
\text { simulation }\end{array}$ & $\begin{array}{l}\text { Nonparticipants were twice as likely to receive } \\
\text { disability pensions (outcome) than participants, and } \\
\text { even more if the pension was received for mental } \\
\text { disorders. Simulation excluding participants with } \\
\text { a similar profile to non-participants reduced the } \\
\text { association between common mental disorders and the } \\
\text { outcome }\end{array}$ \\
\hline 18 & Manjer et al. 30 & $\begin{array}{c}\text { To investigate the effect of non- } \\
\text { participation on cancer incidence and } \\
\text { mortality }\end{array}$ & $\begin{array}{l}\text { Secondary data, } \\
\text { mailed health } \\
\text { survey }\end{array}$ & Survival model & $\begin{array}{l}\text { Non-participants presented lower cancer incidence } \\
\text { prior to recruitment and higher cancer incidence } \\
\text { during recruitment. The proportion of participants in } \\
\text { the cohort reporting better health was higher than in } \\
\text { the mailed survey }\end{array}$ \\
\hline 19 & $\begin{array}{c}\text { Barchielli \& } \\
\text { Balzi } 25\end{array}$ & $\begin{array}{c}\text { To analyze the effect on mortality of } \\
\text { non-response in a smoking prevalence } \\
\text { survey }\end{array}$ & Secondary data & $\begin{array}{l}\text { Poisson model, } \\
\text { life table } \\
\text { method }\end{array}$ & $\begin{array}{c}\text { All causes mortality was significantly higher among } \\
\text { non-respondents, with higher risks for smoking } \\
\text { related causes }\end{array}$ \\
\hline 20 & $\begin{array}{l}\text { Bergman et } \\
\text { al. } 51\end{array}$ & $\begin{array}{c}\text { To analyze the consequences of } \\
\text { attrition in three years after baseline in } \\
\text { the PART study }\end{array}$ & $\begin{array}{l}\text { Baseline } \\
\text { information, } \\
\text { sample of non- } \\
\text { respondents }\end{array}$ & Logistic model & $\begin{array}{l}\text { Variables associated with non-participation - low } \\
\text { income and education, non-Nordic origin and marital } \\
\text { status - were related with depressive mood as well } \\
\text { in the first wave }\end{array}$ \\
\hline 21 & $\begin{array}{c}\text { Petersen et } \\
\text { al. } 52\end{array}$ & $\begin{array}{l}\text { To investigate wether terminally } \\
\text { ill patients' reported quality-of-life } \\
\text { scores should be adjusted for non- } \\
\text { participation bias }\end{array}$ & $\begin{array}{c}\text { Baseline } \\
\text { information }\end{array}$ & $\begin{array}{l}\text { Imputation } \\
\text { methods for } \\
\text { missing data }\end{array}$ & $\begin{array}{l}\text { Significant underestimation of symptoms in } 4 \text { out of } \\
30 \text { comparisons suggest that imputation of quality- } \\
\text { of-life scores of non-participants in palliative care is } \\
\text { biased based on the available predictors }\end{array}$ \\
\hline
\end{tabular}

(continues) 
Table 2 (continued)

\begin{tabular}{|c|c|c|c|c|c|}
\hline Id & Reference & Objectives * & Data source & Analysis & Results \\
\hline 22 & Rao et al. 53 & $\begin{array}{l}\text { To propose a method based on } \\
\text { propensity scores to analytically } \\
\text { reduce bias due to non-response }\end{array}$ & Secondary data & $\begin{array}{l}\text { Propensity } \\
\text { score based } \\
\text { on baseline } \\
\text { information } \\
\text { and data } \\
\text { imputation }\end{array}$ & $\begin{array}{c}\text { Among the respondents, there was a higher } \\
\text { frequency of women, Caucasian, married and younger } \\
\text { people. Differences due to the proposed weighting } \\
\text { scheme were small }\end{array}$ \\
\hline 23 & Haring et al. 54 & $\begin{array}{l}\text { To determine attrition predictors } \\
\text { and evaluate the effect of extensive } \\
\text { recruitment procedures on attrition } \\
\text { and bias }\end{array}$ & $\begin{array}{l}\text { Baseline } \\
\text { information }\end{array}$ & Logistic model & $\begin{array}{l}\text { The main predictors for attrition were late recruitment } \\
\text { at baseline, unemployment, low educational level, } \\
\text { female gender, and smoking. However attrition bias } \\
\text { was not associated with health-related indicators }\end{array}$ \\
\hline 24 & $\begin{array}{l}\text { Van Loon et } \\
\text { al. } 31\end{array}$ & $\begin{array}{l}\text { To investigate possible response } \\
\text { bias in prevalence estimation and } \\
\text { association measures }\end{array}$ & $\begin{array}{l}\text { Baseline } \\
\text { information }\end{array}$ & Logistic model & $\begin{array}{l}\text { Respondents, as compared to non-respondents, } \\
\text { presented higher socioeconomic status, better } \\
\text { subjective health and healthier behaviors. The } \\
\text { association measures were similar in respondents and } \\
\text { the entire population source }\end{array}$ \\
\hline 25 & $\begin{array}{l}\text { Drivsholm et } \\
\text { al. } 55\end{array}$ & $\begin{array}{l}\text { To compare participants at the } \\
\text { 20-year follow-up study with non- } \\
\text { participants, and to investigate the } \\
\text { representativeness of both groups in } \\
\text { relation to the population source }\end{array}$ & Secondary data & Logistic model & $\begin{array}{c}\text { Participation decreased to } 65 \% \text { in the } 20 \text { th follow-up } \\
\text { year, when non-participants had lower socioeconomic } \\
\text { status, worse health profile and higher mortality rate } \\
\text { than participants }\end{array}$ \\
\hline 26 & Jackson et al. 16 & $\begin{array}{l}\text { To compare participants with complete } \\
\text { clinical examinations to those with just } \\
\text { home interview in the the ARIC study }\end{array}$ & $\begin{array}{l}\text { Baseline } \\
\text { information }\end{array}$ & $\begin{array}{l}\text { Frequencies } \\
\text { comparison }\end{array}$ & $\begin{array}{l}\text { Response rates was similar for white participants, both } \\
\text { male and female, and in all study centers. In general, } \\
\text { respondents presented higher socioeconomic status } \\
\text { and health, but differences were smaller for women }\end{array}$ \\
\hline 27 & $\begin{array}{l}\text { Veenstra et } \\
\text { al. } 29\end{array}$ & $\begin{array}{l}\text { To assess association between health } \\
\text { status at baseline and nonresponse; to } \\
\text { analyze survival in a 5-year follow-up }\end{array}$ & Secondary data & Logistic model & $\begin{array}{c}\text { Among respondents, prevalence of coronary heart } \\
\text { disease was higher. However, their mortality was lower } \\
\text { than noncontacts }\end{array}$ \\
\hline 28 & Young et al. 56 & $\begin{array}{l}\text { To describe factors associated with } \\
\text { attrition in a longitudinal study with } \\
\text { three age cohorts of women }\end{array}$ & $\begin{array}{l}\text { Baseline } \\
\text { information }\end{array}$ & Logistic model & $\begin{array}{l}\text { Variables associated with loss-to-follow-up were: } \\
\text { education (lower), non-English-speaking origin, } \\
\text { current smoker, poorer health and difficulty managing } \\
\text { their income, varying according to cohort age }\end{array}$ \\
\hline 29 & $\begin{array}{l}\text { Caetano et } \\
\text { al. } 57\end{array}$ & $\begin{array}{l}\text { To identify characteristics of non- } \\
\text { respondents in a survey among } \\
\text { couples on violence and drinking }\end{array}$ & Secondary data & Logistic model & $\begin{array}{l}\text { Male non-respondents were younger, less educated, } \\
\text { more often unemployed and drinkers. Among } \\
\text { women, having been an abuse victim during } \\
\text { childhood increased response }\end{array}$ \\
\hline 30 & Garcia et al. 28 & $\begin{array}{l}\text { To evaluate attrition in a Spanish } \\
\text { population-based cohort }\end{array}$ & $\begin{array}{l}\text { Baseline } \\
\text { information }\end{array}$ & Logistic model & $\begin{array}{l}\text { Death and moving to another town were the main } \\
\text { reasons of nonresponse. Refusals were associated } \\
\text { with working status (disabled and retired) and place of } \\
\text { birth (other regions of Spain or in foreign countries); } \\
\text { emigration with civil status, age and education as well }\end{array}$ \\
\hline 31 & Hara et al. 58 & $\begin{array}{l}\text { To examine factors influencing the } \\
\text { recruitment in a study collecting } \\
\text { genetic data }\end{array}$ & $\begin{array}{l}\text { Baseline } \\
\text { information }\end{array}$ & Logistic model & $\begin{array}{l}\text { Sex (male) and age (younger) presented lower } \\
\text { participation rates. The survey location (easy access } \\
\text { to participants' residence) and reminders sent to } \\
\text { subjects significantly improved the participation rate }\end{array}$ \\
\hline 32 & $\begin{array}{c}\text { Kjoller \& } \\
\text { Thoning } 33\end{array}$ & $\begin{array}{l}\text { To analyze trends in nonresponse and } \\
\text { assess bias on morbidity prevalence }\end{array}$ & Secondary data & Logistic model & $\begin{array}{l}\text { Refusals increased } 4.3 \% \text { in seven years (from } 1987 \\
\text { to 1994). Nonrespondents were defined by a } \\
\text { combination of sociodemographic characteristics. } \\
\text { Nonrespondents hospital admission rates were higher } \\
\text { than respondents six months before data collection, } \\
\text { and similar afterwards }\end{array}$ \\
\hline
\end{tabular}

(continues) 
Table 2 (continued)

\begin{tabular}{|c|c|c|c|c|c|}
\hline Id & Reference & Objectives * & Data source & Analysis & Results \\
\hline 33 & $\begin{array}{l}\text { Jacobsen et } \\
\text { al. } 32\end{array}$ & $\begin{array}{l}\text { To evaluate associations between } \\
\text { socioeconomic factors and } \\
\text { participation in the Danish National } \\
\text { Birth Cohort }\end{array}$ & Secondary data & $\begin{array}{l}\text { Frequencies } \\
\text { comparison }\end{array}$ & $\begin{array}{c}\text { Groups with low socioeconomic status were } \\
\text { underrepresented as compared to the background } \\
\text { population }\end{array}$ \\
\hline 34 & $\begin{array}{l}\text { Montgomery et } \\
\text { al. } 59\end{array}$ & $\begin{array}{l}\text { To investigated potential bias due } \\
\text { to non-participation in the follow-up } \\
\text { of a large cohort study on pesticide } \\
\text { applicators }\end{array}$ & Secondary data & Logistic model & $\begin{array}{l}\text { Non-respondents at follow-up were younger, less } \\
\text { educated, with lower body mass index and poorer } \\
\text { health behaviors but better health conditions, and } \\
\text { lower pesticide use. Estimates of exposure-disease } \\
\text { associations did not present strong bias }\end{array}$ \\
\hline 35 & $\begin{array}{l}\text { Jousilahti et } \\
\text { al. } 60\end{array}$ & $\begin{array}{l}\text { To evaluate total and cause specific } \\
\text { mortality comparing participants cohort } \\
\text { study }\end{array}$ & Secondary data & Survival model & $\begin{array}{c}\text { At eight year follow up, mortality of non-participating } \\
\text { men and women was higher than participating, } \\
\text { except for smoking related causes }\end{array}$ \\
\hline 36 & May et al. 61 & $\begin{array}{l}\text { To evaluate potential predictors } \\
\text { of non-response that are available } \\
\text { at baseline (socio-economic- } \\
\text { demographic, health, )follow-up } \\
\text { duration and contact strategies }\end{array}$ & $\begin{array}{l}\text { Baseline } \\
\text { information }\end{array}$ & Logistic model & $\begin{array}{l}\text { Age (younger), sex (male), marital status (single), } \\
\text { poorer health conditions, and undernourishment or } \\
\text { obesity were associated with non-response }\end{array}$ \\
\hline 37 & Batty \& Gale 62 & $\begin{array}{l}\text { To investigated variables associated } \\
\text { with non-response and its impact on } \\
\text { the association measures of several } \\
\text { known risk factors and cardiovascular } \\
\text { mortality }\end{array}$ & Secondary data & Survival model & $\begin{array}{l}\text { The non-participants had higher CVD mortality than } \\
\text { participants. However, the association measures } \\
\text { between the risk factors evaluated and the mortality } \\
\text { was not affected by non-response }\end{array}$ \\
\hline 38 & Dugue et al. 63 & $\begin{array}{l}\text { To estimate excess mortality } \\
\text { comparing participants and non- } \\
\text { participants in cervical screening }\end{array}$ & Secondary data & Survival model & $\begin{array}{l}\text { All cause mortality and HPV-related mortality was } \\
\text { higher for non-participants in cervical screening, and } \\
\text { the hazard ratio increased over time }\end{array}$ \\
\hline 39 & Hara et al. 23 & $\begin{array}{l}\text { To evaluate the healthy volunteer } \\
\text { effect comparing mortality } \\
\text { rates among respondents and } \\
\text { nonrespondents }\end{array}$ & Secondary data & Poisson model & $\begin{array}{l}\text { Mortality was higher among nonrespondents for } \\
\text { all causes studied, although with different effects } \\
\text { according do sex. The relative risk varied as well } \\
\text { according to the length of follow-up }\end{array}$ \\
\hline 40 & $\begin{array}{l}\text { Benfante et } \\
\text { al. } 64\end{array}$ & $\begin{array}{l}\text { To investigate differences between } \\
\text { participants and nonparticipants and } \\
\text { the potential introduction of bias in the } \\
\text { association measures }\end{array}$ & Secondary data & $\begin{array}{l}\text { Frequencies } \\
\text { comparison }\end{array}$ & $\begin{array}{l}\text { Total mortality, cancer mortality, and coronary heart } \\
\text { disease incidence rates were higher in non-examined } \\
\text { men, but the differences decreased over time. No } \\
\text { bias was found }\end{array}$ \\
\hline 41 & Ferrie et al. 24 & $\begin{array}{l}\text { To evaluate association between } \\
\text { nonresponse at baseline and } \\
\text { missing follow-up contacts and } \\
\text { general mortality, and mortality by } \\
\text { socioeconomic position }\end{array}$ & Secondary data & Survival model & $\begin{array}{l}\text { Non-response at baseline and at any follow-up } \\
\text { contact was associated with doubling the mortality } \\
\text { hazard }\end{array}$ \\
\hline 42 & François et al. 65 & $\begin{array}{l}\text { To demonstrate how it is possible } \\
\text { to obtain a satisfactory rate of } \\
\text { participation in a cohort study, } \\
\text { and to compare participants and } \\
\text { nonparticipants }\end{array}$ & $\begin{array}{c}\text { Baseline } \\
\text { information }\end{array}$ & $\begin{array}{l}\text { Frequencies } \\
\text { comparison }\end{array}$ & $\begin{array}{l}\text { The main factors associated with the response rate } \\
\text { were: linguistic region, age, income, civil status, } \\
\text { educational and alcohol/drugs consumption }\end{array}$ \\
\hline 43 & Walker et al. 22 & $\begin{array}{l}\text { To compare the mortality rates and } \\
\text { the demographic characteristics } \\
\text { between participants and } \\
\text { nonparticipants }\end{array}$ & Secondary data & $\begin{array}{l}\text { Frequencies } \\
\text { comparison }\end{array}$ & $\begin{array}{l}\text { Non-participants were younger, more likely to be } \\
\text { unmarried and work in less skilled jobs. Their mortality } \\
\text { rates were higher in the first three years of follow-up, } \\
\text { decreasing afterwards. CVD mortality was similar in } \\
\text { both groups }\end{array}$ \\
\hline 44 & David et al. 66 & $\begin{array}{l}\text { To assess the performance of two } \\
\text { different models with two end points } \\
\text { each, in analyzing loss-to-follow-up }\end{array}$ & Secondary data & $\begin{array}{l}\text { Logistic and } \\
\text { survival model }\end{array}$ & $\begin{array}{l}\text { Survival models performed better than logistic } \\
\text { models }\end{array}$ \\
\hline
\end{tabular}

(continues) 
Table 2 (continued)

\begin{tabular}{|c|c|c|c|c|c|}
\hline Id & Reference & Objectives * & Data source & Analysis & Results \\
\hline 45 & Froom et al. 67 & $\begin{array}{l}\text { To investigate the healthy volunteer } \\
\text { effect in an occupationally cohort of } \\
\text { male industrial employees }\end{array}$ & Secondary data & Survival model & $\begin{array}{c}\text { All cause mortality hazard ratio was higher in } \\
\text { nonparticipants, and the difference persisted up to } 8 \\
\text { years of follow-up }\end{array}$ \\
\hline 46 & Bopp et al. 68 & $\begin{array}{l}\text { To evaluate feasibility and quality of } \\
\text { linkage procedure in providing follow- } \\
\text { up information }\end{array}$ & Secondary data & Survival model & $\begin{array}{l}\text { Linkage success was independent of any variables. } \\
\text { Losses in } 10 \text { years were } 4.7 \% \text {. Participants of the study } \\
\text { had lower mortality than the general population }\end{array}$ \\
\hline 47 & Criqui et al. 69 & $\begin{array}{l}\text { To evaluate differences in } \\
\text { cardiovascular health status according } \\
\text { to participation in a population based } \\
\text { study }\end{array}$ & $\begin{array}{l}\text { Baseline } \\
\text { information, } \\
\text { non-respondents } \\
\text { telephone } \\
\text { interview }\end{array}$ & $\begin{array}{l}\text { Frequencies } \\
\text { comparison }\end{array}$ & $\begin{array}{l}\text { Non-respondents presented more CVD but did not } \\
\text { differ on known hypertension. Impact on prevalence } \\
\text { estimates was small due to low proportion of non- } \\
\text { response }\end{array}$ \\
\hline 48 & $\begin{array}{l}\text { Lindsted et } \\
\text { al. } 70\end{array}$ & $\begin{array}{l}\text { To assess the healthy volunteer effect } \\
\text { comparing mortality rates between the } \\
\text { respondents to a small questionnaire } \\
\text { with respondents to a full detailed } \\
\text { questionnaire }\end{array}$ & Secondary data & Survival model & $\begin{array}{l}\text { Hazard ratio for different mortality causes was larger } \\
\text { for non-respondents, but the difference decreased } \\
\text { over time }\end{array}$ \\
\hline 49 & $\begin{array}{c}\text { Thygesen et } \\
\text { al. } 71\end{array}$ & $\begin{array}{l}\text { To estimate the effect of drop-out on } \\
\text { the association between alcohol intake } \\
\text { and mortality }\end{array}$ & Secondary data & Poisson model & $\begin{array}{l}\text { Loss to-follow-up was associated with increased } \\
\text { mortality and incidence rates of heart disease, some } \\
\text { cancers, and liver diseases related to alcohol intake }\end{array}$ \\
\hline 50 & $\begin{array}{c}\text { Vestbo \& } \\
\text { Rasmussen } 72\end{array}$ & $\begin{array}{l}\text { To evaluate if baseline characteristics } \\
\text { could provide sufficient information } \\
\text { about non-response bias }\end{array}$ & Secondary data & Logistic model & $\begin{array}{l}\text { At baseline, respondents and non-respondents } \\
\text { presented similar profiles (smoking, lung function and } \\
\text { respiratory symptoms). However, non-respondents } \\
\text { had larger rates of hospital admission due to } \\
\text { respiratory diseases, indicating that equal baseline } \\
\text { profile does not protect against non-response bias }\end{array}$ \\
\hline
\end{tabular}

CVD: cardiovascular diseases

* Objectives presented here were the most related to the objective of this review.

ated with participation, in spite of its importance in some of them $11,18,19,20,21,22,23,24,25$.

Study region showed no association with participation, in spite of the diversity of places evaluated. Participating in studies voluntarily, giving time, information and biological material is all related to ideas of social capital and volunteering 16 , and we expected variation according to local cultural components.

Participation in studies has also been associated with behavioral variables and with general state of health. Non-participants report greater consumption of alcohol, smoking and poor general state of health 12,15,2019,21,22,23,26,27,28,29,30,31,32, 33,34. This information, however, are not available in most publications, limiting the scope of our study.

Strategies to increase participation proportion have been proposed in terms of persuading individuals who are reluctant or hesitant; however, willingness to participate is not always accompanied by commitment to adhere to the study in the long term ${ }^{35}$. Lastly, we agree with the argument of
Morton et al. 3 that more information should be requested on the profile of participation and its potential bias.

There is a major need to pursue methodological studies to evaluate the impacts of non-participation on measures of effect in cohort studies. Strategies for that kind of evaluation include comparing participants with non-participants through administrative data bases (sex, age, place of residence), application of summary questionnaires and passive follow-up of eligible population to evaluate mortality 4 . Recent publications from journals with high impact factors show that nonparticipation is mostly ignored or dismissed by many authors, although some are attempting to reduce it or mention it as a limitation in their study 36 .

In conclusion, our findings suggest that the drive for participation and compliance should be assessed previously to funding the cohort study, and specific local knowledge should be included in addressing the potential participants. 
Figure 2

Correlation of year the baseline year and participation rate.
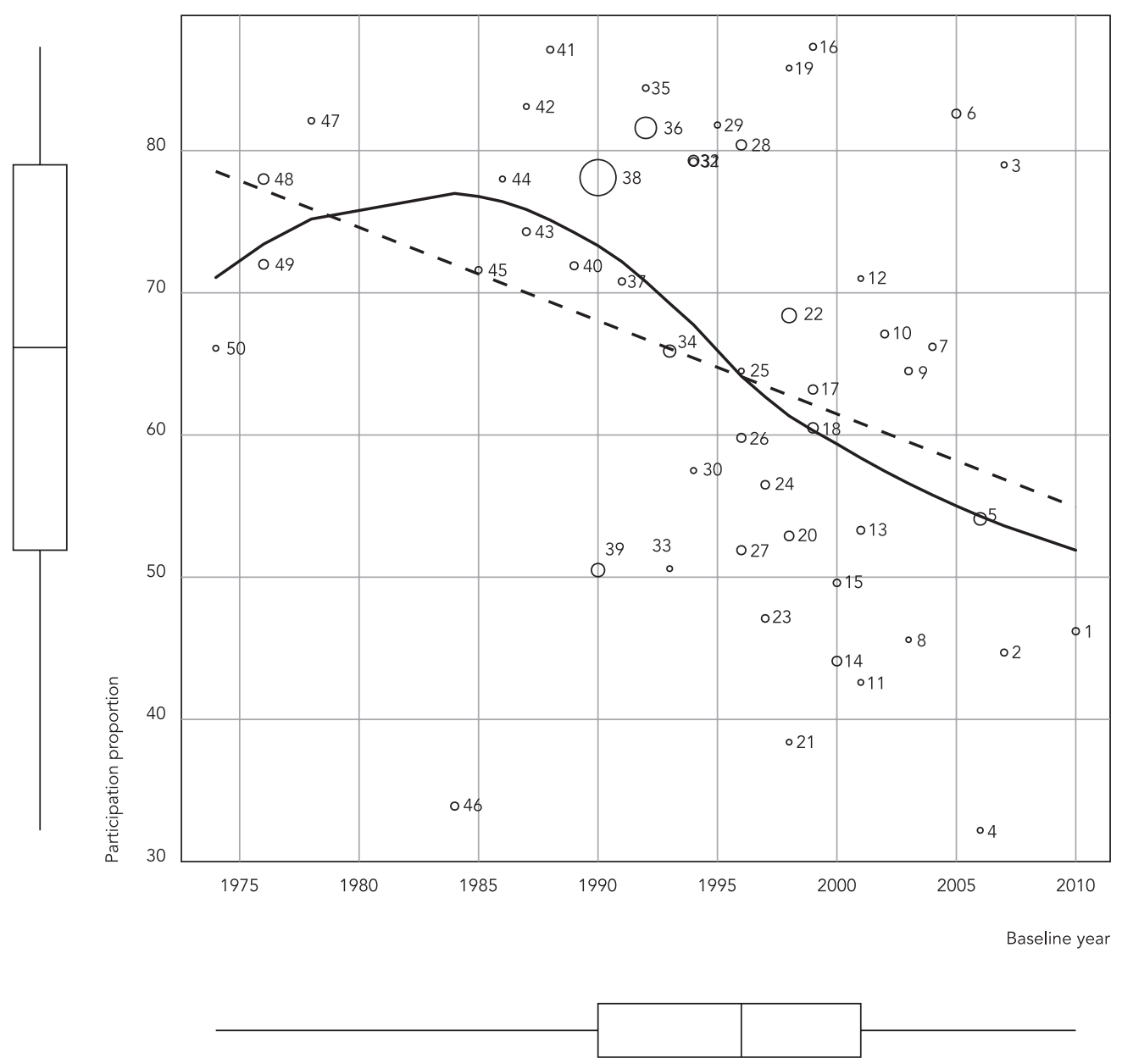
Univariate and multiple meta-regression models.

\begin{tabular}{|c|c|c|c|c|c|c|c|}
\hline \multirow[t]{2}{*}{ Variables } & \multirow[t]{2}{*}{ VAF } & \multicolumn{3}{|c|}{ Univariate meta-regression models } & \multicolumn{3}{|c|}{$\begin{array}{l}\text { Multiple meta-regression models } \\
\qquad V A F=18.1 \%\end{array}$} \\
\hline & & OR & $95 \% \mathrm{Cl}$ & $\mathrm{p}$-value & OR & $95 \% \mathrm{Cl}$ & p-value \\
\hline Age (mean) & $2.6 \%$ & 0.99 & $0.96-1.01$ & 0.24 & 0.97 * & $0.95-1.00$ & 0.04 \\
\hline Proportion of women & $6.7 \%$ & 1.01 & $0.99-1.03$ & 0.21 & 1,01 & $0.99-1.03$ & 0.18 \\
\hline Baseline year & $11.9 \%$ & 0.97 & $0.95-0.99$ & 0.01 & 0.97 * & $0.94-0.99$ & 0.02 \\
\hline \multicolumn{8}{|l|}{ Selection (baseline: Sampling) } \\
\hline Census & $0.0 \%$ & 0.99 & $0.66-1.48$ & 0.95 & - & - & - \\
\hline \multicolumn{8}{|l|}{ Population (baseline: General } \\
\hline \multicolumn{8}{|l|}{ population) } \\
\hline Other & $0.0 \%$ & 1.02 & $0.63-1.67$ & 0.93 & - & - & - \\
\hline \multicolumn{8}{|c|}{ Outcomes (baseline: General health) } \\
\hline Cardiovascular diseases & $3.6 \%$ & 0.78 & $0.49-1.24$ & 0.30 & - & - & - \\
\hline Other & & 1.12 & $0.70-1.80$ & 0.65 & - & - & - \\
\hline \multicolumn{8}{|c|}{ Study region (baseline: Northern Europe) } \\
\hline U.S.A. & $7.5 \%$ & 1.17 & $0.67-2.04$ & 0.59 & 0.94 & $0.51-1.73$ & 0.85 \\
\hline Continental Europe & & 0.69 & $0.43-1.12$ & 0.13 & 0.64 & $0.38-1.07$ & 0.09 \\
\hline Other & & 1.10 & $0.65-1.89$ & 0.72 & 0.94 & $0.52-1.69$ & 0.83 \\
\hline
\end{tabular}

95\% Cl: 95\% confidence interval; OR: odds ratio; VAF: variance accounted for.

* For the change of one unit in the variable causes decline the odds of participation.

\section{Resumen}

La proporción de no participación en estudios de cohorte se asocia también con la exposición y probabilidad de ocurrencia de hechos que pueden generar sesgos en las estimaciones de interés. El objetivo de este estudio es realizar una revisión sistemática y un metaanálisis de artículos que describen la participación en estudios de cohortes y evaluar las características asociadas con la participación. Una revisión sistemática fue realizada (MEDLINE, Scopus y Web of Science), en busca de artículos que describen la relación de participación basada en estudios de cohortes. Se seleccionaron 2964 artículos, de los cuales se identificaron preliminarmente 50. Entre estos, la proporción promedio de participación fue de un 64,7\%. Utilizando la metarregresión, sólo la edad, años de referencia y la región de estudio (borderline) se asociaron con la participación. Teniendo en cuenta la disminución de la participación en los últimos años, y el coste de los estudios de cohortes, es esencial buscar información para evaluar el potencial de la no participación antes de comprometer recursos.

Sesgo de Selección; Estudios de Cohortes; Métodos Epidemiológicos

\section{Contributors}

S. H. A. Silva Junior was responsible for the first draft of the manuscript and data analyzes and contributed to the conception and design of the study. S. M. Santos, C. M. Coeli and M. S. Carvalho contributed to the conception and design of the study. All authors contributed significantly to interpreting the results, commented extensively on subsequent revisions, and read and approved the final manuscript.

\section{Acknowledgments}

We acknowledge Wolfgang Viechtbauer (at Maastricht University, Maastricht, Netherlands) for his help with the R metafor package, and Israel Souza for revising the selected paper. C. M. Coeli was supported by research fellowship grants from CNPq (304101/2011-7) and Faperj (E26/102.771/2012). 


\section{References}

1. Kelsey JL. Methods in observational epidemiology. New York: Oxford University Press; 1996.

2. Greenland S. Response and follow-up bias in cohort studies. Am J Epidemiol 1977; 106:184-7.

3. Morton LM, Cahill J, Hartge P. Reporting participation in epidemiologic studies: a survey of practice. Am J Epidemiol 2006; 163:197-203.

4. Stang A. Nonresponse research: an underdeveloped field in epidemiology. Eur J Epidemiol 2003; 18:929-31.

5. Galea S, Tracy M. Participation rates in epidemiologic studies. Ann Epidemiol 2007; 17:643-53.

6. Higgins JPT, Green S. Cochrane handbook for systematic reviews of interventions. Volume 5. Chichester: Wiley-Blackwell; 2008.

7. Moher D, Liberati A, Tetzlaff J, Altman DG; The PRISMA Group. Preferred reporting items for systematic reviews and meta-analyses: the PRISMA Statement. PLoS Med 2009; 6:e1000097.

8. De Ridder MAJ, Stijnen T, Hokken-Koelega ACS. A new method to determine mean adult height from incomplete follow-up data. Horm Res 2007; 67:205-10.

9. United Nations Statistics Division. Composition of macro geographical (continental) regions, geographical sub-regions, and selected economic and other groupings. https://unstats.un.org/unsd/ methods $/ \mathrm{m} 49 / \mathrm{m} 49$ regin.htm (accessed on $01 /$ Mar/2013).

10. Viechtbauer W. Conducting meta-analyses in $\mathrm{R}$ with the metafor package. J Stat Softw 2010; 36(3). http://www.jstatsoft.org/v36/i03/paper.

11. Bopp M, Braun J, Faeh D. Variation in mortality patterns among the general population, study participants, and different types of nonparticipants: evidence from 25 years of follow-up. Am J Epidemiol 2014; 180:1028-35.

12. Harald K, Salomaa V, Jousilahti P, Koskinen S, Vartiainen E. Non-participation and mortality in different socioeconomic groups: the FINRISK population surveys in 1972-92. J Epidemiol Community Health 2007; 61:449-54.

13. Carlsson F, Merlo J, Lindström M, Ostergren P-O, Lithman T. Representativity of a postal public health questionnaire survey in Sweden, with special reference to ethnic differences in participation. Scand J Public Health 2006; 34:132-9.

14. Lundberg I, Damström Thakker K, Hällström T, Forsell Y. Determinants of non-participation, and the effects of non-participation on potential cause-effect relationships, in the PART study on mental disorders. Soc Psychiatry Psychiatr Epidemiol 2005; 40:475-83.

15. Jackson R, Chambless LE, Yang K, Byrne T, Watson $\mathrm{R}$, Folsom A, et al. Differences between respondents and nonrespondents in a multicenter community-based study vary by gender ethnicity. The Atherosclerosis Risk in Communities (ARIC) Study Investigators. J Clin Epidemiol 1996; 49:1441-6.
16. Stang A, Moebus S, Dragano N, Beck EM, Möhlenkamp S, Schmermund A, et al. Baseline recruitment and analyses of nonresponse of the Heinz Nixdorf Recall Study: identifiability of phone numbers as the major determinant of response. Eur J Epidemiol 2005; 20:489-96.

17. Lissner L, Skoog I, Andersson K, Beckman N, Sundh V, Waern M, et al. Participation bias in longitudinal studies: experience from the Population Study of Women in Gothenburg, Sweden. Scand J Prim Health Care 2003; 21:242-7.

18. Boshuizen HC, Viet AL, Picavet HSJ, Botterweck A, van Loon AJM. Non-response in a survey of cardiovascular risk factors in the Dutch population: determinants and resulting biases. Public Health 2006; 120:297-308.

19. Knudsen AK, Hotopf M, Skogen JC, Overland S, Mykletun A. The health status of nonparticipants in a population-based health study: the Hordaland Health Study. Am J Epidemiol 2010; 172:1306-14.

20. Goldberg M, Chastang JF, Zins M, Niedhammer I, Leclerc A. Health problems were the strongest predictors of attrition during follow-up of the GAZEL cohort. J Clin Epidemiol 2006; 59:1213-21.

21. Walker M, Shaper AG, Cook DG. Non-participation and mortality in a prospective study of cardiovascular disease. J Epidemiol Community Health 1987; 41:295-9.

22. Hara M, Sasaki S, Sobue T, Yamamoto S, Tsugane S. Comparison of cause-specific mortality between respondents and nonrespondents in a populationbased prospective study: ten-year follow-up of JPHC Study Cohort I. Japan Public Health Center. J Clin Epidemiol 2002; 55:150-6.

23. Ferrie JE, Kivimäki M, Singh-Manoux A, Shortt A, Martikainen P, Head J, et al. Non-response to baseline, non-response to follow-up and mortality in the Whitehall II cohort. Int J Epidemiol 2009; 38:831-7.

24. Barchielli A, Balzi D. Nine-year follow-up of a survey on smoking habits in Florence (Italy): higher mortality among non-responders. Int J Epidemiol 2002; 31:1038-42.

25. Putnam RD. Tuning in, tuning out: the strange disappearance of social capital in America. PS Polit Sci Polit 1995; 28:664-83.

26. Shahar E, Folsom AR, Jackson R. The effect of nonresponse on prevalence estimates for a referent population: insights from a population-based cohort study. Atherosclerosis Risk in Communities (ARIC) Study Investigators. Ann Epidemiol 1996; 6:498-506.

27. Garcia M, Fernandez E, Schiaffino A, Borrell C, Marti M, Borras JM. Attrition in a populationbased cohort eight years after baseline interview: The Cornella Health Interview Survey Follow-up (CHIS.FU) Study. Ann Epidemiol 2005; 15:98-104. 
28. Veenstra MY, Friesema IHM, Zwietering PJ, Garretsen HFL, Knottnerus JA, Lemmens PHHM. Lower prevalence of heart disease but higher mortality risk during follow-up was found among nonrespondents to a cohort study. J Clin Epidemiol 2006; 59:412-20.

29. Manjer J, Carlsson S, Elmståhl S, Gullberg B, Janzon L, Lindström M, et al. The Malmö Diet and Cancer Study: representativity, cancer incidence and mortality in participants and non-participants. Eur J Cancer Prev 2001; 10:489-99.

30. Van Loon AJM, Tijhuis M, Picavet HSJ, Surtees PG, Ormel J. Survey non-response in the Netherlands: effects on prevalence estimates and associations. Ann Epidemiol 2003; 13:105-10.

31. Jacobsen TN, Nohr EA, Frydenberg M. Selection by socioeconomic factors into the Danish National Birth Cohort. Eur J Epidemiol 2010; 25:349-55.

32. Kjøller M, Thoning H. Characteristics of non-response in the Danish Health Interview Surveys, 1987-1994. Eur J Public Health 2005; 15:528-35.

33. Carter KN, Imlach-Gunasekara F, McKenzie SK, Blakely T. Differential loss of participants does not necessarily cause selection bias. Aust N Z J Public Health 2012; 36:218-22.

34. Forthofer RN. Investigation of nonresponse bias in NHANES II. Am J Epidemiol 1983; 117:507-15.

35. Groves RM, Peytcheva E. The impact of nonresponse rates on nonresponse bias: a meta-analysis. Public Opin Q 2008; 72:167-89.

36. Keeble C, Barber S, Law GR, Baxter PD. Participation bias assessment in three high-impact journals. SAGE Open 2013; 3(4):2158244013511260.

37. Studer J, Baggio S, Mohler-Kuo M, Dermota P, Gaume J, Bertholet N, et al. Examining non-response bias in substance use research: are late respondents proxies for non-respondents? Drug Alcohol Depend 2013; 132:316-23.

38. Kaerlev L, Kolstad HA, Hansen AM, Thomsen JF, Kærgaard A, Rugulies R, et al. Are risk estimates biased in follow-up studies of psychosocial factors with low base-line participation? BMC Public Health 2011; 11:539.

39. Langley JD, Lilley R, Wilson S, Derrett S, Samaranayaka A, Davie G, et al. Factors associated with non-participation in one or two follow-up phases in a cohort study of injured adults. Inj Prev 2013; 19:428-33.

40. Alkerwi A, Sauvageot N, Couffignal S, Albert A, Lair M-L, Guillaume M. Comparison of participants and non-participants to the ORISCAV-LUX population-based study on cardiovascular risk factors in Luxembourg. BMC Med Res Methodol 2010; 10:80.

41. Langhammer A, Krokstad S, Romundstad P, Heggland J, Holmen J. The HUNT study: participation is associated with survival and depends on socioeconomic status, diseases and symptoms. BMC Med Res Methodol 2012; 12:143.
42. Eriksson A-K, Ekbom A, Hilding A, Ostenson C-G. The influence of non-response in a populationbased cohort study on type 2 diabetes evaluated by the Swedish Prescribed Drug Register. Eur J Epidemiol 2012; 27:153-62.

43. Osler M, Kriegbaum M, Christensen U, Holstein B, Nybo Andersen A-M. Rapid report on methodology: does loss to follow-up in a cohort study bias associations between early life factors and lifestyle-related health outcomes? Ann Epidemiol 2008; 18:422-4

44. Buckley B, Murphy AW, Glynn L, Hennigan C. Selection bias in enrollment to a programme aimed at the secondary prevention of ischaemic heart disease in general practice: a cohort study. Int J Clin Pract 2007; 61:1767-72.

45. Schmidt CO, Raspe H, Pfingsten M, Hasenbring M, Basler HD, Eich W, et al. Does attrition bias longitudinal population-based studies on back pain? Eur J Pain 2011; 15:84-91.

46. Martikainen PT, Valkonen T. Excess mortality of unemployed men and women during a period of rapidly increasing unemployment. Lancet 1996; 348:909-12.

47. Holden L, Ware RS, Passey M. Characteristics of nonparticipants differed based on reason for nonparticipation: a study involving the chronically ill. J Clin Epidemiol 2008; 61:728-32.

48. Taylor AW, Dal Grande E, Gill T, Chittleborough CR, Wilson DH, Adams RJ, et al. Do people with risky behaviours participate in biomedical cohort studies? BMC Public Health 2006; 6:11.

49. Alonso A, Seguí-Gómez M, de Irala J, Sánchez-Villegas A, Beunza JJ, Martínez-Gonzalez MA. Predictors of follow-up and assessment of selection bias from dropouts using inverse probability weighting in a cohort of university graduates. Eur J Epidemiol 2006; 21:351-8.

50. Bergman P, Ahlberg G, Forsell Y, Lundberg I. Nonparticipation in the second wave of the PART study on mental disorder and its effects on risk estimates. Int J Soc Psychiatry 2010; 56:119-32.

51. Petersen MA, Pedersen L, Groenvold M. Does nonparticipation in studies of advanced cancer lead to biased quality-of-life scores? J Palliat Med 2009; 12:1023-8.

52. Rao RS, Sigurdson AJ, Doody MM, Graubard BI. An application of a weighting method to adjust for nonresponse in standardized incidence ratio analysis of cohort studies. Ann Epidemiol 2005; 15:12936.

53. Haring R, Alte D, Völzke H, Sauer S, Wallaschofski H, John U, et al. Extended recruitment efforts minimize attrition but not necessarily bias. J Clin Epidemiol 2009; 62:252-60.

54. Drivsholm T, Eplov LF, Davidsen M, Jørgensen T, Ibsen H, Hollnagel H, et al. Representativeness in population-based studies: a detailed description of non-response in a Danish cohort study. Scand J Public Health 2006; 34:623-31. 
55. Young AF, Powers JR, Bell SL. Attrition in longitudinal studies: who do you lose? Aust N Z J Public Health 2006; 30:353-61.

56. Caetano R, Ramisetty-Mikler S, McGrath C. Characteristics of non-respondents in a US national longitudinal survey on drinking and intimate partner violence. Addict 2003; 98:791-7.

57. Hara M, Higaki Y, Imaizumi T, Taguchi N, Nakamura K, Nanri H, et al. Factors influencing participation rate in a baseline survey of a genetic cohort in Japan. J Epidemiol 2010; 20:40-5.

58. Montgomery MP, Kamel F, Hoppin JA, Beane Freeman LE, Alavanja MCR, Sandler DP. Effects of selfreported health conditions and pesticide exposures on probability of follow-up in a prospective cohort study. Am J Ind Med 2010; 53:486-96.

59. Jousilahti P, Salomaa V, Kuulasmaa K, Niemelä M, Vartiainen E. Total and cause specific mortality among participants and non-participants of population based health surveys: a comprehensive follow up of 54372 Finnish men and women. J Epidemiol Community Health 2005; 59:310-5.

60. May AM, Adema LE, Romaguera D, Vergnaud A-C, Agudo A, Ekelund U, et al. Determinants of nonresponse to a second assessment of lifestyle factors and body weight in the EPIC-PANACEA study. BMC Med Res Methodol 2012; 12:148.

61. Batty GD, Gale CR. Impact of resurvey non-response on the associations between baseline risk factors and cardiovascular disease mortality: prospective cohort study. J Epidemiol Community Health 2009; 63:952-5.

62. Dugué P-A, Lynge E, Rebolj M. Mortality of nonparticipants in cervical screening: register-based cohort study. Int J Cancer 2014; 134:2674-82.

63. Benfante R, Reed D, MacLean C, Kagan A. Response bias in the Honolulu Heart Program. Am J Epidemiol 1989; 130:1088-100.
64. François Y, Truan P, Gmel G. Response rate and analysis of non-responses in a cohort study. Soz Praventivmed 1997; 42:186-91.

65. David MC, van der Pols JC, Williams GM, Alati R, Green AC, Ware RS. Risk of attrition in a longitudinal study of skin cancer: logistic and survival models can give different results. J Clin Epidemiol 2013; 66:888-95.

66. Froom P, Melamed S, Kristal-Boneh E, Benbassat J, Ribak J. Healthy volunteer effect in industrial workers. J Clin Epidemiol 1999; 52:731-5.

67. Bopp M, Braun J, Faeh D, Gutzwiller F; Swiss National Cohort Study Group. Establishing a followup of the Swiss MONICA participants (1984-1993): record linkage with census and mortality data. BMC Public Health 2010; 10:562.

68. Criqui MH, Barrett-Connor E, Austin M. Differences between respondents and non-respondents in a population-based cardiovascular disease study. Am J Epidemiol 1978; 108:367-72.

69. Lindsted KD, Fraser GE, Steinkohl M, Beeson WL. Healthy volunteer effect in a cohort study: temporal resolution in the Adventist Health Study. J Clin Epidemiol 1996; 49:783-90.

70. Thygesen LC, Johansen C, Keiding N, Giovannucci E, Grønbaek M. Effects of sample attrition in a longitudinal study of the association between alcohol intake and all-cause mortality. Addict 2008; 103:1149-59.

71. Vestbo J, Rasmussen FV. Baseline characteristics are not sufficient indicators of non-response bias follow up studies. J Epidemiol Community Health 1992; 46:617-9.

Submitted on 04/Sep/2014

Final version resubmitted on 08/May/2015

Approved on 21/May/2015 